\title{
An Optimized Scheme to Generating Support Structure for 3D Printing
}

\author{
Mao Yu-xin ${ }^{1(\bowtie)}$, Wu Li-fang ${ }^{1,2}$, Qiu Jian-kang ${ }^{1}$, and Wei Runyu ${ }^{1}$ \\ ${ }^{1}$ Beijing University of Technology, Beijing 100124, China \\ suika2333@sina.com, Ifwu@bjut.edu.cn \\ 2 Beijing Engineering Research Center of 3D Printing \\ for Digital Medical Health, Beijing, China \\ lfwu@bjut.edu.cn
}

\begin{abstract}
This paper presents an improved algorithm to generating support structure for 3D printing model. Firstly, the candidate regions are obtained from the shadow area in each layer. Secondly, the maximal random points sampling in anchor map is implemented by the Poisson disk sampling. Thirdly, the support structure is generated in each layer according to the sampling points for the 3D model. The experimental results compared with state-of-the-art algorithm show that the proposed algorithm can save about 5-20\% support sticks.
\end{abstract}

Keywords: 3D printing, support structure $\cdot$ Poisson disk sampling

\section{Introduction}

With the explosion development of 3D printing technology, many kinds of 3D models can be printed. In most cases, the support structure is needed for a printable model. In another word, the support structure is the hands and the feet for a printable model. Without those, the model is hard to be printed. The support structure will be removed and throw away from the printed model. It is part of the waste materials, and the less the support structure, the better. So, the evaluation of the support structure includes cost of printing materials and stability.

By now, there are many kinds of algorithms to generating support structure. These algorithms can be divided into two classes: model-based and slice-based. The main idea of model-based algorithm is to generate the support structure from analysis of the 3D model directly, the typical algorithms include scaffolding structure [1] and tree bracing structure [2]. The structure generation of model-based has a very intuitive effect to observe whether the structure is suitable and easy to reconstitute the model. But the disadvantage of the model-based methods is that the support structure is generated manually, which costs much time. About the slice-based algorithm, the typical algorithm based on slicing is IFTFSIS (Intersection-Free and Topologically Faithful Slicing of Implicit Solid) [3]. As its name suggests, it uses the layers of the model after slicing

This paper is supported by key project of Beijing Municipal Science and Technology Commission with grant number K2101311201401. 
to generate the support structure automatically. This method can avoid processing the model in 3D, and easily optimize the support structure where the areas need. Also it is able to adapt to the different models automatically.

IFTFSIS uses 2D image to solve the problem in 3D space. According to the layered manufacturing, it overlaps the binary images of each layer and gets an integrated area where support structure needs to be added. The maximal covering criterion [4] is used to get the positions where support sticks are added. IFTFSIS transfers the support structure from $3 \mathrm{D}$ to $2 \mathrm{D}$ image, it is accurate and effective. But the initial support structure is generated by equal space sampling, which is not easy to cover the region efficiently. In this paper, we propose an improved IFTFSIS algorithm to use Poisson disk sampling to replace equal space sampling. The simulated experimental results show that the proposed scheme can save about 5-20\% material of support structure.

\section{The Proposed Scheme}

\subsection{Intersection-Free and Topologically Faithful Slicing of Implicit Solid}

Intersection-Free and Topologically Faithful Slicing of Implicit Solid was proposed by Huang [3] in 2014. It includes three steps: To generate the candidate regions from sliced layers; To generate the support points in sampling, and to obtain the support structure.

The 3D model is firstly sliced layer by layer, and each layer is presented as an image. Then the difference image of two neighboring layers is obtained by image subtraction. After superimposing all extracted area, an image named "anchor map" can be obtained. Then "close" operation is practiced o fill gaps in the image. The final image is shown in Fig. 1 (a).

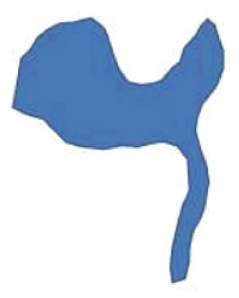

(a)

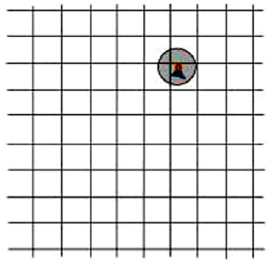

(d)

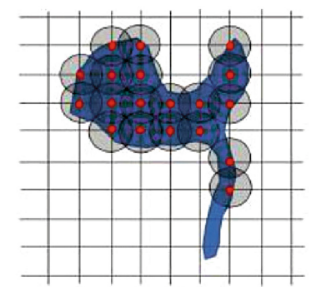

(b)

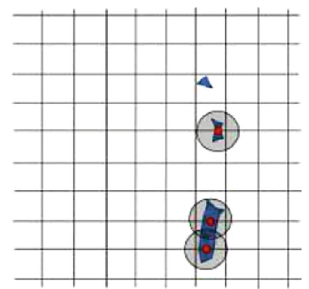

(c)

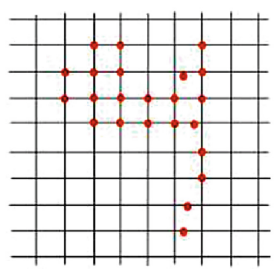

(e)

Fig. 1. Illustration of IFTFSIS [3] (Color figure online) 
The uniform sampling is used to intersect with the anchor map (Fig. 1 (b)) and exclude the anchor-support region (anchor-support region is a set of circles of which the centers are support points) (Fig. 1 (c)). Then check if the remaining area overlap the grid lines, if it happens, choose that area and draw the anchor-support region. Finally, traverse the whole image to find remaining area and draw the anchor-support region again (Fig. 1 (d)). All red points in Fig. 1 (e) are the place where the supporting sticks need to be added.

After the above work, use these points which are on the anchor map to find the ways to connect every point in order to generate a support structure in scaffolding structure.

\subsection{The Proposed Scheme}

\subsubsection{The Framework of the Proposed Scheme}

In the improved scheme, the support structure is also obtained from the slices of the 3D model. The framework of the scheme is shown in Fig. 2. Firstly, the candidate regions are obtained from the shadow area in each layer. Secondly, the maximal random points sampling in anchor map is implemented by the Poisson disk sampling. Thirdly, the support structure is generated in each layer according to the sampling points for the $3 \mathrm{D}$ model.

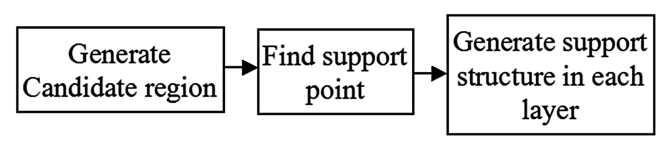

Fig. 2. Flow chart of support structure generation

\subsubsection{Why Poisson Disk Sampling Is Needed}

Poisson disk sampling is a kind of random sampling with certain conditions [5]. In 2D image, it is used to generate a natural distribution about the artificial landscape. By Poisson disk sampling, a set of sampling points could be generated with the following properties:

(1) They are tightly packed together;

(2) They are not closer to each other than a specified minimum distance.

Figure 3 shows a pair of compared the random sampling and Poisson disk sampling. From Fig. 3 (b), we can observe that the distance between each pair of sampling points is not shorter than a constant, $r$, which also means that each point is used to draw a circle with the radius, $r$, so that at most one point is in each circle (also called disk). This method not only satisfies the maximal covering criterion, but also decreases the amount of the points in the image as much as possible. The following formulas are the basic conditions about Poisson disk sampling: 


$$
\begin{gathered}
\text { Bias - free : } \forall x_{i} \in X, \forall \Omega \subset D_{i-1} ; P\left(x_{i} \in \Omega\right)=\frac{\operatorname{Area}(\Omega)}{\operatorname{Area}\left(D_{i-1}\right)} \\
\text { Empty disk : } \forall x_{i}, x_{j} \in X, x_{i} \neq x_{j}:\left\|x_{i}-x_{j}\right\| \geq r \\
\text { Maximal : } \forall x \in D, \exists x_{i} \in X:\left\|x-x_{i}\right\|<r
\end{gathered}
$$

where $X=\left\{x_{i}\right\}$ is the random set of points; $D$ is the given domain; $r$ is the disk radius. With the above three formulas, they can satisfy: (1) bias free; (2) one disk only have one point inside; (3) try to make the minimum amount of points, and also ensure the maximal covering.

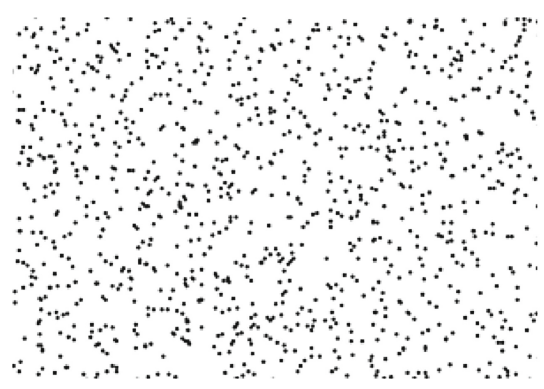

(a) Random sampling

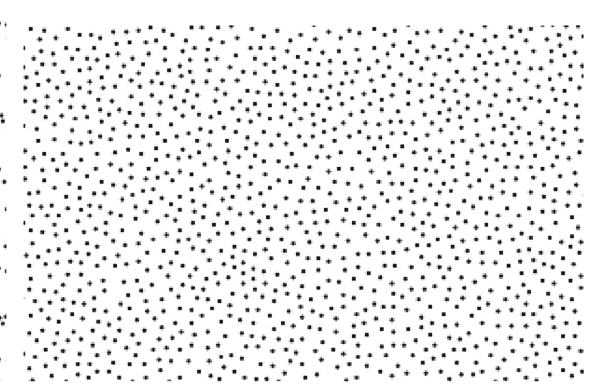

(b) Poisson sampling

Fig. 3. Comparison of Random sampling and Poisson disk sampling

\subsubsection{The Poisson Disk Sampling Algorithm}

The Poisson disk sampling algorithm includes 5 steps:

(1) Create the anchor map and background image with the square grid;

(2) Do the Poisson disk sampling on the background.

(3) Make two images, anchor map and background, become same size and scan two images together. If the corresponding pixel value is equal to 0 (in this case, target is black), record this point and use it to draw the circle on anchor map. Continue the scanning, and repeat the above work, until the last pixel of the image.

(4) Find the area which is not overlapped with circles in anchor map, then put a new point in it. Continue the scanning, and repeat the above work, until the whole anchor map are overlapped with the circles.

(5) Extract the points being retained, and then use them to create the support sticks.

In this paper, the "throw dart method" [6] is used to separate whole candidate region into many square grid. Firstly, we generate a background square grid, each square is called a cell. The diagonal in each cell equals to $r$, which can ensure that each cell only can contain one point (except the boundary of cells). Next, pick one cell at random and throw the dart (point) into it, the position of the dart in the cell is random too. After that, we remove this cell and repeat the above work in $n$ times ( $n$ is not less than 500) with 
the constraints that the distance between any two darts is not shorter than $r$. Then with the darts as the centers and $r$ as the radius, draw circles and try to overlap the whole background. Finally we traverse whole background to find if there are any small areas which are not be overlapped by the circles. If there are, chose any points in those small areas and draw the circles, and repeat the detection, until completely cover the background. Illustrations are shown in Fig. 4 (a) - (d).

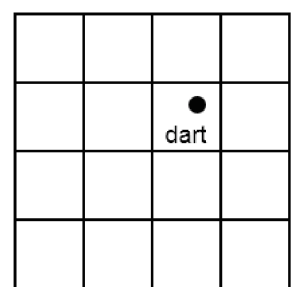

(a)

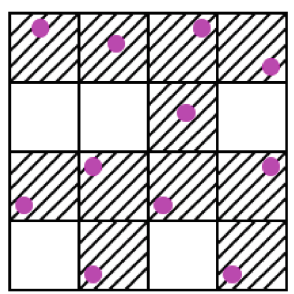

(c)

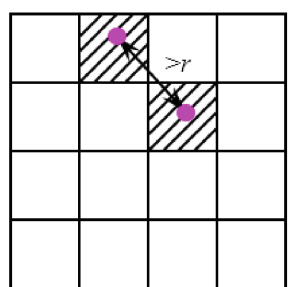

(b)

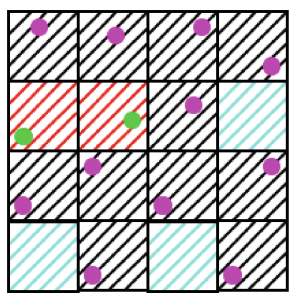

(d)

Fig. 4. Illustrations of the throw dart method; (a) Throw the first dart; (b) The second dart needs to ensure: 1) Different cells, 2) The distance of two darts are not shorter than $r$; (c) Continue throwing with $n$ times; (d) Scan whole background and find whether there any cell are not be chosen. The blue cells mean that there are no dart satisfies Eq. (2), because the whole cells are overlapped by neighboring circles where center point is in neighboring cells. (Color figure online)

\section{Experiments}

In order to compare the distributions of support points of IFTFSIS [3] and the improved scheme, 6 models are used, as shown in Fig. 5. We generate the support structure using IFTFSIS and ours respectively. The support points are compared in Table 1.

From Table 1 we can see that the proposed algorithm need less support points for all of the example models. It can save about average $13.31 \%$ waste materials compared with the traditional IFTFSIS.

We further check the distribution of support points. Figure 6 shows the corresponding results (from IFTFSIS or Ours). It is obvious that the support points from 
Table 1. Comparisons of support structure

\begin{tabular}{l|l|l|l|l|l|l|l}
\hline & (a) & (b) & (c) & (d) & (e) & (f) & Ave \\
\hline Ref [3] & 62 & 27 & 42 & 172 & 104 & 59 & \\
\hline Ours & 52 & 23 & 39 & 144 & 100 & 48 & \\
\hline Percentage of saved waste material (\%) & 16.13 & 14.81 & 7.14 & 16.28 & 3.85 & 18.64 & 13.31 \\
\hline
\end{tabular}

The average run-time is $600 \mathrm{~ms}$ of this work.

IFTFSIS are with equal space (as shown in Fig. 6 (a0)-(a6)). But the support points from the proposed scheme distribute randomly in Fig. 6 (b0)-(b6). Sometimes, because of the random of the sampling points, if the anchor map has long and narrow area, such as in Fig. 5 (c) and (e), the advantage of the results will not be so obvious, as shown in Table 1, the less waste materials will be saved, such as $7.14 \%$ for Fig. 5 (c) and $3.85 \%$ for Fig. 5 (e).

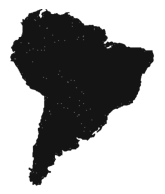

(a)

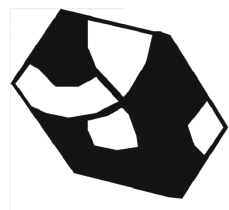

(d)

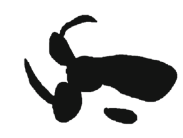

(b)

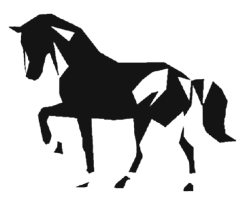

(c)

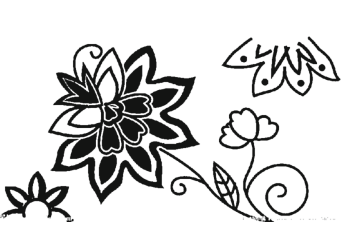

(e)

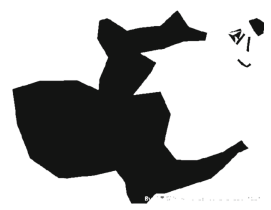

(f)

Fig. 5. The candidate regions of some example models.

In IFTFSIS, the resulted support points are stable because of the uniformed grid. However, in our algorithm, Poisson disk sampling is a random sampling and sometimes, the sampling points vary each time.

In IFTFSIS there are a lot of overlapped areas. But In our algorithm, Poisson disk sampling causes the as less as possible overlapped areas. But long narrow area limits the randomness of Poisson disk sampling. It reduces the difference between Poisson disk sampling method and uniformed grid method. 


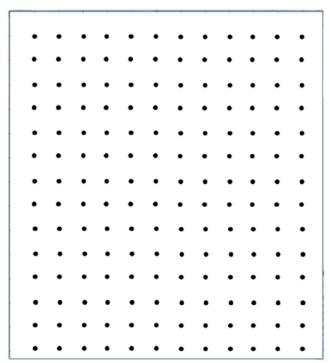

(a0)

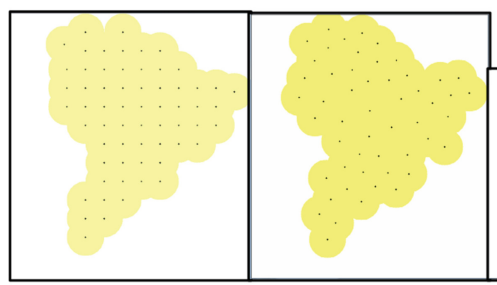

(a1)

(b 1)

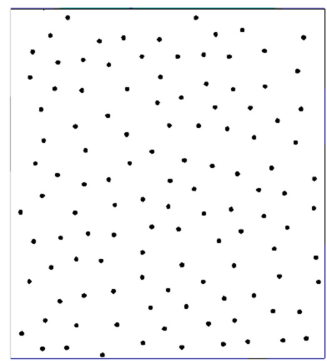

(b0)

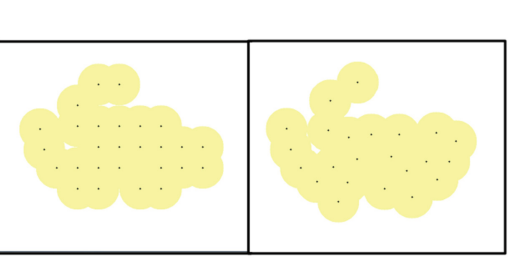

(a2)

(b2)

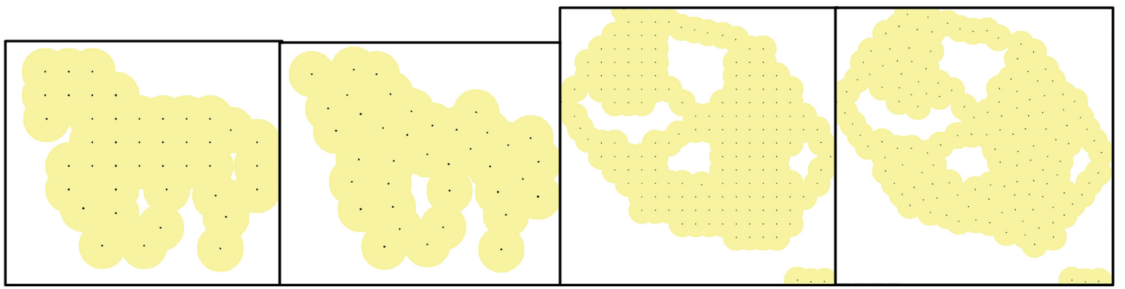

(a3)

(b3)

(a4)

(b4)

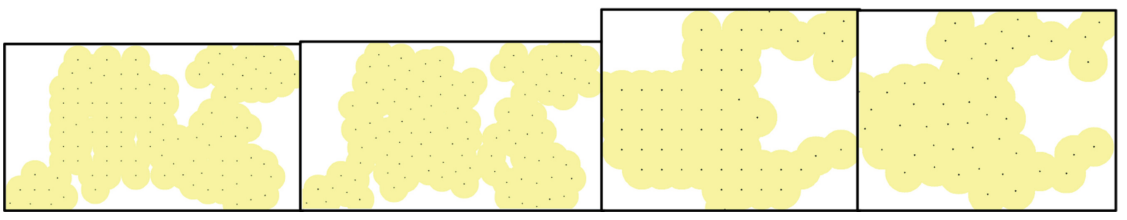

(a5)

(b5)

(a6)

(b6)

Fig. 6. Comparison of support points. (a0) the uniform grid sampling from IFTFSIS in a square region; (b0) a Poisson disk sampling model in a square region; (a1-a6) are the results from IFTFSIS for the models in Fig. 5 and (b1-b6) are the results from ours for the models in Fig. 5.

\section{Conclusions}

This paper presents an improved algorithm to generating support structure for printing 3D model. It decreases the amount of the supporting sticks about $13 \%$ because Poison disk sampling method is used.

How to reduce the amount of the support structure does not still have the most optimal solution in theory. This paper uses square grid model to do the Poisson disk 
sampling, and obtain good results. In addition, another new Poisson disk sampling with regular hexagon grid may reduce the amount of structure and calculation time at the same time. In the future, we will simplify support structure with the regular hexagon grid.

\section{References}

1. Dumas, J., Hergel, J., Lefebvre, S.: Bridging the gap: automated steady scaffoldings for $3 \mathrm{~d}$ printing [J]. ACM Trans. Graphics (TOG) 33(4), 98 (2014)

2. Vanek, J., Galicia, J.A.G., Benes, B.: Clever support: efficient support structure generation for digital fabrication [C]. Computer Graphics Forum 33(5), 117-125 (2014)

3. Huang, P., Wang, C.C.L., Chen, Y.: Intersection-Free and topologically faithful slicing of implicit solid [J]. J. Comput. Inf. Sci. Eng. 13(2), 021009 (2013)

4. Church, R., Velle, C.R.: The maximal covering location problem [J]. Pap. Reg. Sci. 32(1), 101-118 (1974)

5. Ebeida, M.S., Mitchell, S.A., Patney, A. et al.: A simple algorithm for maximal poisson-disk sampling in high dimensions [C]. In: Computer Graphics Forum, 31(2pt4), pp. 785-794. Blackwell Publishing Ltd (2012)

6. White, K.B., Cline, D., Egbert, P.K.: Poisson disk point sets by hierarchical dart throwing [C]. In: IEEE Symposium on Interactive Ray Tracing, 2007. RT 2007, pp. 129-132. IEEE (2007) 